\title{
Melanopsin-expressing retinal ganglion cell loss and behavioral analysis in the Thy1-CFP-DBA/2J mouse model of glaucoma
}

\author{
ZHANG Qi ${ }^{1,2,3}$, VUONG Helen ${ }^{4,5,6}$, HUANG Xin ${ }^{1,2,3}$, WANG YanLing ${ }^{1,2,3}$, \\ BRECHA Nicholas C. ${ }^{4,5,6}$, PU MingLiang ${ }^{1,2,3} \&$ GAO Jie ${ }^{1,2,3^{*}}$ \\ ${ }^{1}$ Department of Anatomy, School of Basic Medical Sciences, Peking University, Beijing 100191, China; \\ ${ }^{2}$ Key Laboratory on Machine Perception (Ministry of Education), Peking University, Beijing 100191, China; \\ ${ }^{3}$ Key Laboratory for Visual Impairment and Restoration (Ministry of Education), Peking University, Beijing 100191, China; \\ ${ }^{4}$ Department of Neurobiology and Molecular, Cellular, and Integrative Physiology, University of California, Los Angeles, CA 90095, USA; \\ ${ }^{5}$ Jules Stein Eye Institute, University of California, Los Angeles, CA 90095, USA; \\ ${ }^{6}$ UCLA College of Life Sciences, University of California, Los Angeles, CA 90095, USA
}

Received April 5, 2013; accepted April 29, 2013; published online May 30, 2013

\begin{abstract}
In this study, the role of melanopsin-expressing retinal ganglion cells (mRGCs) in the glaucoma-induced depressive behavioral response pattern was investigated. The CFP-D2 transgenic glaucoma animal model from five age groups was used in this study. Immunohistochemical labeling, quantitative analysis of mRGC morphology, open field test (OFT), and statistical analysis were used. In comparison with C57 BL/6 mice, the age-matched CFP-D2 mice had significantly elevated intraocular pressure (IOP). We observed parallel morphological changes in the retina, including a reduction in the density of cyan fluorescent protein(CFP) expressing cells (cells $\mathrm{mm}^{-2}$ at 2 months of age, $1309 \pm 26$; 14 months, $878 \pm 30, P<0.001$ ), mRGCs $(2$ months, $48 \pm 3 ; 14$ months, 19 $\pm 4, P<0.001)$, Brn3b-expressing RGCs ( 2 months, 1283 $\pm 80 ; 14$ months, $950 \pm 31, P<0.001)$, Brn-3b expressing mRGCs (5 months, $50.17 \% \pm 5.5 \%$; 14 months, $12.61 \% \pm 3.8 \%, P<0.001$ ), and reduction in the dendritic field size of mRGCs $\left(\mathrm{mm}^{2}\right.$ at 2 months, $0.077 \pm 0.015 ; 14$ months, $\left.0.065 \pm 0.015, P<0.05\right)$. CFP-D2 mice had hyperactive locomotor activity patterns based on OFT findings of the total distance traveled, number of entries into the center, and time spent in the center of the testing apparatus. The glaucoma induced hyperactive response pattern could be associated with dysfunctional mRGCs, most likely Brn-3b-positive mRGCs in CFP-D2 mice.
\end{abstract}

pigmentary glaucoma, Thy1-CFP-DBA/2J mouse, retinal ganglion cells, melanopsin-expressing retinal ganglion cells, depression

Citation: Zhang Q, Vuong H, Huang X, et al. Melanopsin-expressing retinal ganglion cell loss and behavioral analysis in the Thy 1-CFP-DBA/2J mouse model of glaucoma . Sci China Life Sci, 2013, 56: 720-730, doi: 10.1007/s11427-013-4493-1

Glaucoma, a progressive retinal degenerative disease, is the second leading cause of blindness worldwide, with a disproportionate bias towards women and Asians [1,2]. Pigmentary glaucoma is caused by disruption of the posterior iris by the lens zonule, leading to melanin pigment accumulation within the anterior segment structures and the trabecular meshwork, subsequently blocking aqueous outflow.

*Corresponding author (email: gaojie@ bjmu.edu.cn)
Blockage of the aqueous outflow results in elevated intraocular pressure (IOP), a consistent risk factor for glaucoma. High IOP then causes progressive peripheral to central death of retinal ganglion cells (RGCs) and their axons [3].

Previous clinical studies have shown a high prevalence of depression, cognitive impairment, anxiety [4-14], and altered mood symptoms in patients with age-related eye diseases. Loss of RGCs results in loss of melanopsinexpressing RCGs (mRGCs) and axonal innervation of the 
hypothalamic nuclei and basal limbic centers, including the suprachiasmatic nucleus (SCN), paraventricular nucleus (PVN), subparaventricular zone, ventrolateral preoptic area (VLPO), and medial amygdaloid nucleus (mMA) [15]. Indeed, in addition to regulating circadian photoentrainment, mRGCs are also involved in the pupillary light reflex $[16,17]$. Recent findings also suggest that mRGCs are part of a neurocircuit that modulates memory and behavioral mood regulation $[18,19]$, and therefore it is possible that loss of mRGCs can affect behavioral response patterns.

Currently, many rodent glaucoma models are generated by a variety of techniques to elevate IOP or produce acute physical insults to RGC axons. In particular, RGC axotomy is induced by intraorbital optic nerve crush [20], optic nerve transection [21], elevated IOP-induced transient ischemia [22], or by selective ligature of the ophthalmic vessels [23]. Nevertheless, these animal models do not offer the clinically observed glaucomatous optic neuropathy (GON) of progressive degeneration of the RGC population. Recently there are glaucomatous animal models generated by specific genetic mutations, for instance, alteration of the serine protease (PRSS56) induced angle-closure glaucoma in mice [24]. The DBA/2J mouse, by contrast, is a well-established model of spontaneous pigmentary glaucoma. This mouse strain is characterized by age related elevation of IOP, progressive death of RGCs, and atrophic excavation of the optic nerve. These pathological characteristics closely resemble human glaucoma [25-27]. A Thy1-CFP D2 (CFP-D2) line was created by congenitally breeding the DBA/2J line (The Jackson Laboratory, Bar Harbor, ME, USA) with the Thy1-CFP line [28,29]. The Thy1-CFP mouse line transgenically labels the RGCs by a Thy 1 promoter controlled cyan fluorescent protein (CFP). To better understand the relationship between GON-induced RGC degenerative morphology and GON-induced behavioral changes, our study characterized morphological alterations in conventional RGCs (cRGCs) and mRGCs, and evaluated behavior at multiple ages in CFP-D2 mice compared to C57BL/6 mice. Because the morphological properties of cRGCs in glaucoma retinas have been extensively studied, this current study concentrated on characterizing mRGCs. To quantitatively investigate morphological properties of mRGCs, we analyzed dendritic field area and dendritic branch pattern. Open field test (OFT) was used to assess behavioral characteristics of rodents [30,31]. Thus, we measured OFT locomotor activity and anxiety levels of the C57BL/6 and CFP-D2 mice.

\section{Materials and methods}

\subsection{Animals}

The transgenic Thy1-CFP-DBA/2J (CFP-D2) line was created by crossing Thy1-CFP mice, which express CFP in ganglion cells, with DBA/2J mice, which develop progres- sive pigmentary glaucoma. All mice were housed under specific pathogen-free conditions in the Animal Facility of Peking University Health Science Center, with controlled humidity and temperature $\left(24 \pm 1^{\circ} \mathrm{C}\right), 12 \mathrm{~h}$ light/dark conditions (light on at 6:00 a.m.), and with food and water $a d$ libitum. A total of 40 female mice were used in these experiments. Twenty-five were female CFP-D2 transgenic mice (from the University of California, Los Angeles, USA) at $2,5,8,11$, and 14 months of age ( 5 mice/age group), and 15 were control female C57BL/6 mice (Peking University Health Science Center, China) at 2, 8, and 14 months of age (5 mice/age group). In addition, two age groups of older CFP-D2 mice (20 and 24 months of age) were used in the clinical slit-lamp examinations (two animals/group). All experiments were performed in accordance with the Association for Research in Vision and Ophthalmology Statement for the Use of Animals in Ophthalmic and Vision Research.

\subsection{Clinical slit-lamp analysis}

The anterior segments of the CFP-D2 mice, in each age group, were examined using a slit-lamp microscope and photographed with a digital camera at $40 \times$ magnification. All photographs were taken under the same conditions and saved as BMP files. The images were processed with graphic software (Adobe Photoshop CS5, Adobe Systems, Inc., San Jose, CA, USA).

\subsection{IOP measurements}

IOP measurements were obtained from the CFP-D2 mice (2, $5,8,11$, and 14 months of age) and C57BL/6 mice $(2,8$, and 14 months) using the TONO-PEN AVIA VET Tonometer (Reichert, Inc., Depew, NY, USA). Mice were anesthetized using intraperitoneal injections of ketamine $(50 \mathrm{mg}$ $\mathrm{kg}^{-1}$ ) and xylazine $(5 \mathrm{mg} \mathrm{kg}-1)$. The tip of the Tonometer was gently applied toward the center of the cornea, and the measurements were displayed on the liquid crystal displays (LCDs). Once six readings were obtained, the LCD displayed the average IOP in millimeters of mercury $(\mathrm{mm} \mathrm{Hg})$, along with a statistical confidence indicator. To avoid diurnal variations, all IOP measurements were performed between 9:00 and 11:00 a.m.

\subsection{Tissue preparation and whole-mount retina im- munohistochemistry}

Mice were euthanized with an overdose of ketamine and xylazine, the superior pole of each retina was burn-marked for orientation, and the eyes were removed and dissected. After the removal of the cornea, lens, and vitreous body, the retina was removed from the eyecup after 1 min in $4 \%$ paraformaldehyde (Electron Microscopy Sciences \#15710, Ft. Washington, PA, USA), flattened between two slides with 
spacers on both ends, and fixed for an additional $60 \mathrm{~min}$. The retinas were then cryoprotected by immersion in $30 \%$ sucrose solution $/ 0.1 \mathrm{~mol} \mathrm{~L}^{-1}$ phosphate-buffered saline (PBS) at $4^{\circ} \mathrm{C}$ overnight. The next day, the retinas were placed in a 24-well tissue culture dish with gentle agitation on a shaker (unless otherwise stated, the following protocol was carried out on a shaker), and incubated in $1 \%$ sodium borohydride (in deionized water) for $1 \mathrm{~h}$ at room temperature. The free-floating retinas were rinsed six times with 0.1 mol L ${ }^{-1}$ PBS ( $\mathrm{pH}$ 7.4) for 10 min per rinse, and then incubated in a blocking solution containing 10\% normal goat serum, $1 \%$ bovine serum albumin, and $0.5 \%$ Triton $\mathrm{X}-100$ in $0.1 \mathrm{~mol} \mathrm{~L}^{-1} \mathrm{PBS}$ for $2 \mathrm{~h}$ at room temperature. The blocking solution was removed and the primary antibody, diluted in blocking solution, was immediately added to the retinas, and the retinas were incubated for $3-5 \mathrm{~d}$ at $4{ }^{\circ} \mathrm{C}$. After rinsing six times to remove excess primary antibody, the retinas were incubated in secondary antibody for 4-6 $\mathrm{h}$ at room temperature. Following the final washing, the retinas were mounted onto glass slides with the ganglion cell layer up, air-dried, and a cover slip was added using the Fluoro-Gel II Mounting Medium (Electron Microscopy Sciences) containing the fluorescent nuclear dye 4',6-diamidino-2phenylindole (DAPI). As a control for secondary antibody specificity, some retinas were processed in parallel for immunofluorescence staining, with the omission of primary antibody. These specimens showed no detectable signal. The preparations were evaluated using a fluorescence microscopy (BX51, Olympus, Okkonoki, Japan).

\subsection{Antibodies}

The optimal working dilution for each antibody was determined empirically. The primary antibodies and dilutions used in this study were as follows: goat anti-Brn-3b (1:50, SC-6026, Santa Cruz Biotech., Santa Cruz, CA, USA) [32], and polyclonal rabbit anti-melanopsin $(1: 2000$, AB-N38, Advanced Targeting Systems Inc., San Diego, CA, USA) [33]. Immunolabeling with these primary antibodies was visualized using the following secondary antibodies: DyLight 488 and DyLight 549, anti-rabbit IgG (1:1000, Vector Labs, Inc., Burlingame, CA, USA), DyLight 549 rabbit anti-goat IgG $(\mathrm{H}+\mathrm{L})(1: 200$, Kirkegaard \& Perry Labs, Inc., Gaithersburg, MD, USA), and Alexa 594-conjugated donkey anti-goat $\operatorname{IgG}(\mathrm{H}+\mathrm{L})(1: 1000$, Molecular Probes, Grand Island, NY, USA).

\subsection{Cell count and morphologic analysis}

Entirely reconstructed retinal whole mount images taken with the fluorescent microscope (BX51, Olympus) were used to determine the average soma densities of CFP- and Brn-3b-expressing RGCs and mRGCs. Cell counts were determined from confocal reconstructions of the ganglion cell layer images taken from the retinal whole-mounts, and converted to cells $\mathrm{mm}^{-2}$. For CFP- and Brn-3b-expressing RGCs density versus spatial quantification analysis, each retina was divided into superior, inferior, nasal, and temporal quadrants. Four predefined areas $(200 \mu \mathrm{m} \times 200 \mu \mathrm{m} /$ microscope fields), with a separation of $600 \mu \mathrm{m}$, along each quadrant, from the central to peripheral location of the retina, were analyzed, corresponding to approximately $4 \%$ of the total retinal area. The total number of mRGCs in the entire retina was also counted and converted to cells $\mathrm{mm}^{-2}$. CFP fluorescence was used as the primary ganglion cell marker. Retinas were also labeled with Brn-3b to confirm the presence of ganglion cells and cell nuclei. Images of the retina were captured using appropriate filters. The final publication quality images were exported in TIFF format at 300 dpi using Adobe Photoshop CS5 (Adobe Systems, Inc.). For morphologic analysis, the number of primary dendrites of the mRGCs was quantified. For detailed investigation of dendritic patterns of the mRGCs, the dendritic field area and the branches of dendrites of each mRGC were examined using a modification of Sholl's analysis method [34]. Specifically, concentric circles of increasing radius were drawn using the center of the soma as the circle center. The number of dendrites intersecting each circle was counted and plotted against the distance from the center of the soma $(15-150 \mu \mathrm{m})$. The morphology of the dendrites was also investigated in the cross-sectional plane by projecting the stacked images along the $z$-axis. A single-blind approach was used in all cell counting and measurements, to eliminate any subjective bias.

\subsection{Behavior analysis using open field test (OFT)}

The OFT is an experimental method used to assay general locomotor activity and anxiety levels in rodents. In the present study, each mouse (both C57BL/6 and CFP-D2 mice, five animals per age group) was placed in the center point of the open field apparatus (length $74 \mathrm{~cm} \times$ width $74 \mathrm{~cm} \times$ height $40 \mathrm{~cm}$ ). The box was labeled by square crossing, and divided into 16 small grids $(4 \times 4)$. The center four grids refer to the center part of the field. Each mouse was kept in the box for $8 \mathrm{~min}$, and after a 2-min adaptation to the environment, measurements were made, including the total distance traveled, the number of entries into the center, and the time spent in the center/total time spent for the following 6 min. All animals were tested between 18:30 and 20:30. Total motor activity was quantified as the total distance traveled $(\mathrm{mm})$.

\subsection{Statistical analysis}

All data were expressed as mean \pm standard deviation (SD). One-way analysis-of-variance (ANOVA; SPSS 13.0 for Windows, SPSS Inc., Armonk, NY, USA) followed by a post hoc Tukey multiple comparison test were used for each 
analysis. Data analyzed included the IOP of CFP-D2 mice in each age group, cell counts, dendritic field area, and dendritic branching pattern obtained from Sholl's analysis. $P<0.05$ was taken to be statistically significant.

\section{Results}

\subsection{Evaluation of the anterior segment}

The anterior segments of 2- and 5-month-old CFP-D2 mice had normal morphological features. Iris atrophy and pigment dispersion were not detected prior to 8 months of age (Figure 1A). Dispersed pigment and iris atrophy occurred in 8-month-old CFP-D2 mice (Figure 1B) and severely dispersed pigment was observed around the pupillary border in 11-month-old CFP-D2 mice (Figure 1C). Iris atrophy and severity of pigment dispersion increased with age in the CFP-D2 mice. Pigment dispersion was identified throughout the anterior chambers, and keratic precipitates were also present on the cornea (across the surface of the iris) in 14-month-old animals (Figure 1D). Large irregular accumulations of pigmented debris were common in 20-month-old animals (Figure 1E). The iris was severely atrophied and the cornea continued to deteriorate, with the cornea showing calcification and nepheloid zones in 24-month-old animals (Figure 1F). With the development of the glaucoma phenotype, 20- and 24-month-old CFP-D2 mice appeared to have lost the normal features of the anterior segments. These de-

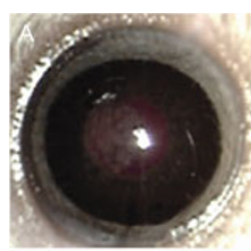

2 months

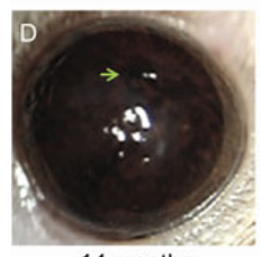

14 months

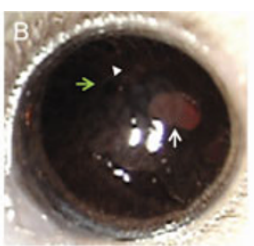

8 months

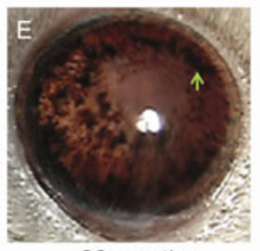

20 months

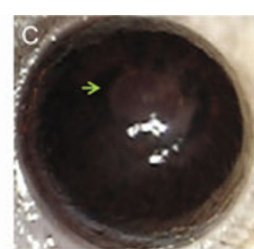

11 months

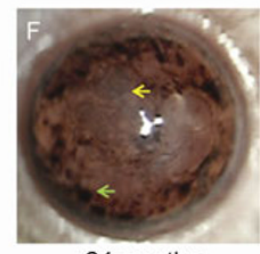

24 months
Figure 1 Clinical appearance of anterior segments and iris morphology in CFP-D2 mice of different ages (slit-lamp analysis, 40x). A, Normal pupil in a 2-month-old CFP-D2 mouse. The pupil was surrounded by a regular iris with a clean edge. The pupil was dilated as the mouse was anesthetized. B, Dispersed pigment (green arrow) and iris atrophy (white arrow) in an 8-month-old mouse. C, Severely dispersed pigment around the pupillary border in an 11-month-old animal (green arrow). D, Pigment dispersion throughout the anterior chambers and keratic precipitates were also present on the cornea, across the surface of the iris, in a 14-month-old animal. E, A largely irregular accumulation of pigmented debris (green arrow) in a 20-month-old animal. F shows a severely atrophied iris, and the cornea was calcified and had nepheloid zones (opacity of cornea, with a translucent iris, and a fixed pupil which exhibits no response to light) (yellow arrow) in a 24-month-old CFP-D2 mouse. teriorating anterior features appeared exclusively in CFP-D2 mice, in comparison with age-matched control animals.

\subsection{Elevation of IOP in CFP-D2 mice}

The age related progressive IOP elevation was determined quantitatively with direct IOP measurements. Fifty eyes from CFP-D2 mice and 30 from controls (C57BL/6 mice) were measured at ages ranging from 2 to 14 months. As shown in Figure 2, IOPs of control animals were stable with age. However, in CFP-D2 mice the average IOP level progressively increased with age from 2 to 11 months (Figure 2). In addition, the IOP level in CFP-D2 mice showed sharp elevations even before the animals reached 11 months of age. In comparison to controls, the elevations of CFP-D2 mice were significantly different in all age groups (2 months of age, (15.2 \pm 0.84$) \mathrm{mm} \mathrm{Hg} ; 5$ months, (17.8 \pm 0.45$) \mathrm{mm} \mathrm{Hg}$; 8 months, $(20.8 \pm 0.45) \mathrm{mm} \mathrm{Hg}, P<0.001)$. Although the elevated IOP appeared to plateau, the elevations were still significantly between 8 and 11 months of age (11 months, $(22.2 \pm 0.84) \mathrm{mm} \mathrm{Hg}, P<0.01)$. However, the elevated IOP showed no significant difference between 11 and 14 months of age (14 months, $(22.2 \pm 0.84) \mathrm{mm} \mathrm{Hg}, P>0.05)$.

\subsection{Changes in CFP expressing RGCs are an indicator of ganglion cell loss in CFP-D2 mice}

Whole mount retinas from CFP-D2 mice (Figure 3A) were processed for quantitative RGC counts. RGC measurements were made from retinas obtained from 2-14-month-old CFP-D2 mice. The average density (number of cells $\mathrm{mm}^{-2}$ ) of CFP-expressing cells in the GCL decreased significantly with age (Figure 3B, 2 months of age, 1309 $\pm 32 ; 5$ months, $1092 \pm 8 ; 8$ months, $1024 \pm 20 ; 11$ months, $953 \pm 37 ; 14$ months,

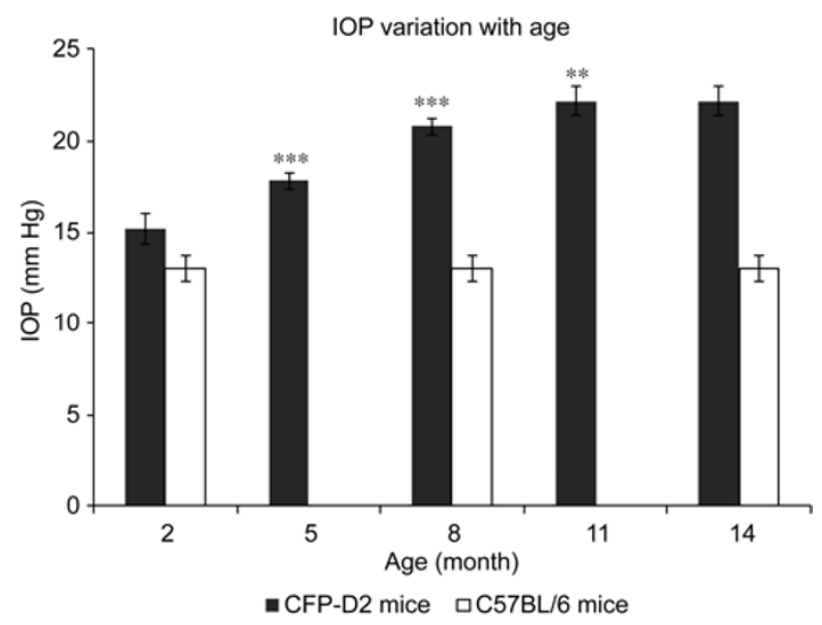

Figure 2 Histogram comparing IOP as a function of age. The age related IOP elevation (mean \pm SD) was present in CFP-D2 mice, whereas IOP did not vary with age in C57BL/6 mice. Comparisons were made in CFP-D2 mice ( 2 vs. 5 months of age, 5 vs. 8 months of age, 8 vs. 11 months of age, and 11 vs. 14 months of age). ${ }^{* *}, P<0.01$; ***, $P<0.001$. 
$878 \pm 34 ; 2$ months of age vs. 5 months, $P<0.001 ; 5$ months vs. 8 months, $P<0.01$; 8 months vs. 11 months, $P<0.01$, and 11 months vs. 14 months, $P<0.01, n=4)$.

\subsection{Loss of Brn3b-expressing cells in the ganglion cell layer (GCL) in CFP-D2 mice}

In both C57BL/6 and CFP-D2 mouse strains, the densities of Brn-3b-positive cells were analyzed from whole-mount retinas obtained from 2-14-month-old mice. There was no significant change in the number of Brn3b-expressing cells in the GCL of C57BL/6 mice from 2-14 months of age (Figure 4E). However, there was variable loss of Brn3bexpressing RGCs in the GCL of the CFP-D2 retinas among the different age groups (Figure $4 \mathrm{~A}-\mathrm{C}$ ). It is evident that Brn3b-expressing cells decreased significantly with age in
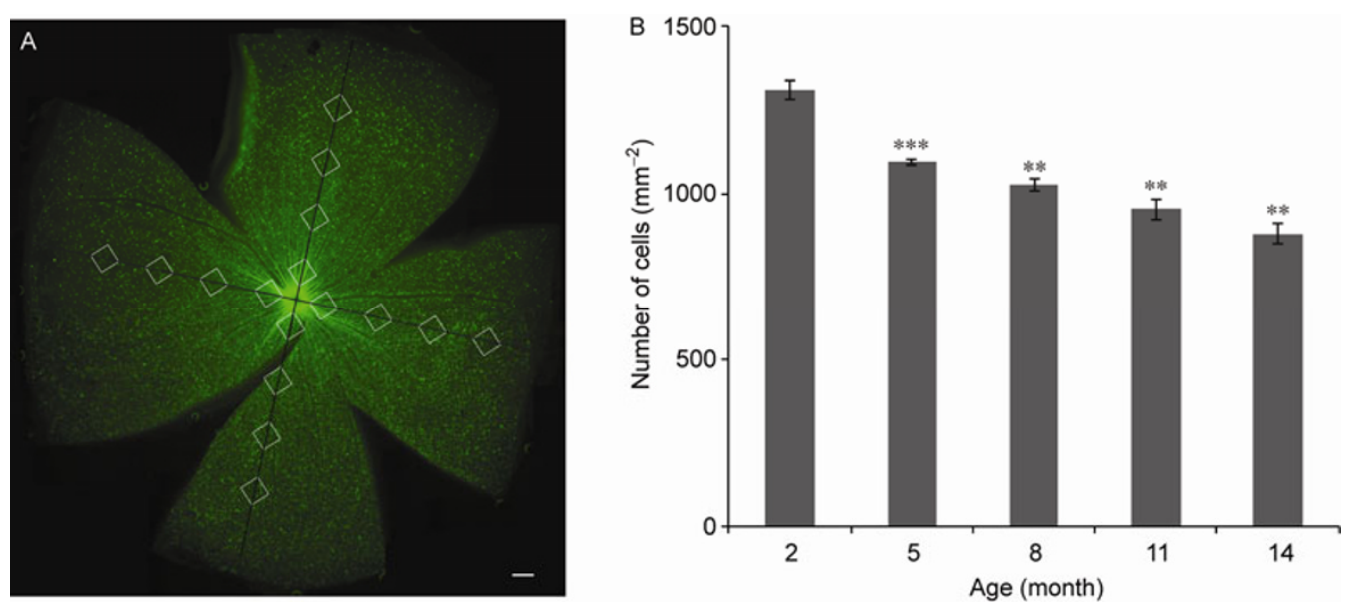

Figure 3 CFP-expressing cell density in CFP-D2 mice. A, CFP-expressing cell distribution pattern in a whole-mount of CFP-D2 mouse retina. Four predefined areas $(200 \mu \mathrm{m} \times 200 \mu \mathrm{m} /$ microscope field area), with an interval of $600 \mu \mathrm{m}$ along each quadrant from the central retina were counted in each retina. B, Histogram comparing average density of CFP-expressing cells in the ganglion cell layer (GCL) (mean \pm SD) as a function of age. Comparisons were made between two age groups ( 2 vs. 5 months old, 5 vs. 8 months old, 8 vs. 1 months old, and 11 vs. 14 months old). Scale bar, $200 \mu$ m. Conventions are the same as used for Figure 2 . **, $P<0.01 ; * *, P<0.001$.
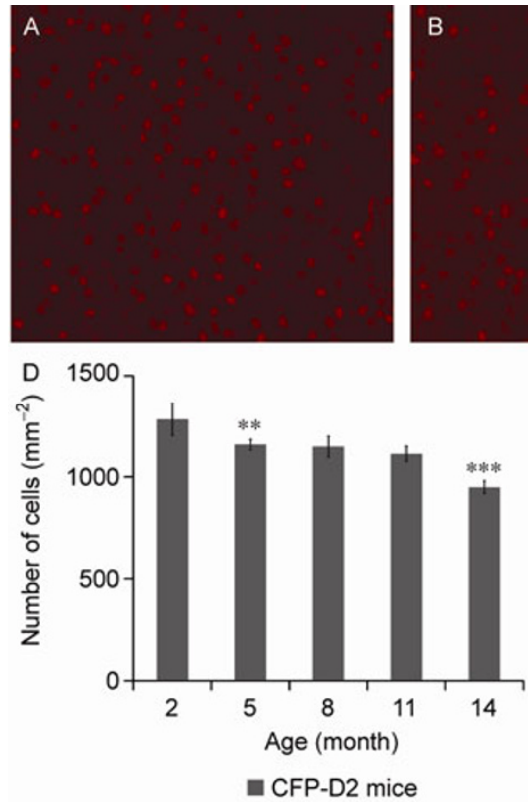
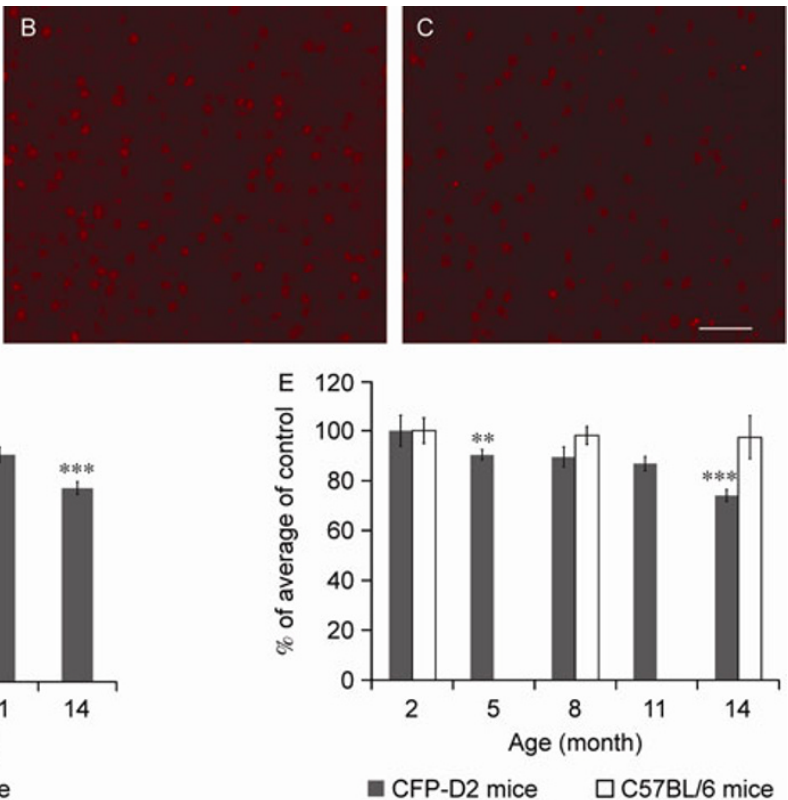

Figure 4 Density distribution patterns of Brn-3b-expressing in RGCs in the GCL of CFP-D2 retina. A-C, Density distribution patterns of Brn-3b-expressing RGCs in retinas of three age groups: 2 months old (A), 8 months old (B), and 14 months old (C). The density distribution of Brn-3b-expressing cells (mean \pm SD) were counted and averaged from four retinas in each age group ( $n=4)$. D, Histogram comparing Brn-3-expressing RGCs in different age groups ( 2 vs. 5 months old, 5 vs. 8 months old, 8 vs. 11 months old, and 11 vs. 14 months old) in CFP-D2 mice. E, Brn-3b-expressing RGCs in CFP-D2 and control animals of different age groups as described above. Scale bar, $50 \mu \mathrm{m}$. $* *, P<0.01 ; * * *, P<0.001$. Conventions are as indicated in Figure 3. 
the CFP-D2 mice ( 2 vs. 5 months of age, $P<0.01, n=4 ; 11$ vs. 14 months of age, $P<0.001, n=4)$. Nevertheless, the differences are insignificant when comparing 5-month-old vs. 8-month-old or 8-month-old vs. 11-month-old CFP-D2 retinas (Figure 4D and E).

\subsection{Morphology of mRGCs in CFP-D2 mice}

The density of mRGCs was quantified in whole mount retinas of CFP-D2 and control mice. The number of mRGCs in C57BL/6 whole-mount retinas did not vary with age $(P>0.05, n=5)$ (Figure 5A-C, G and H). However, the density of mRGCs in CFP-D2 mouse retinas decreased significantly with age, when comparing mRGC counts in 2-month-old versus 11, 14-month-old retinas $(P<0.001$, $n=5)$. However, there was no statistically significant difference between comparisons of similar age groups (2 months vs. 5 months, 5 months vs. 8 months, and 11 months vs. 14 months) $(P>0.05, n=5)$ (Figure 5D-H).

To accurately map the changes in morphology of the mRGCs in CFP-D2 mice, we examined the number of primary dendritic branch points, the dendritic field area, and dendritic branching pattern of these cells. Although dendritic processes may overlap with dendrites of other mRGCs, the dendritic morphology of a well stained $\mathrm{mRGC}$ can be readily identified with high magnification microscope objectives. Using Sholl's analysis, we quantitatively analyzed dendritic morphology and branching patterns of mRGCs. In comparison with mRGCs from 2- and 8-month-old CFP-D2 mice, the average number of dendrites intersecting the circles in 14-month-old mice was substantially reduced $(P<0.05$, $n=10$ cells per retina) (Figure $6 \mathrm{C}$ ).

Because the vast majority of M1s are Brn-3b-negative, this allows us to characterize expression differences between M1s compared to the other types of mRGCs (M2-5) in the CFP-D2 mice. Quantification of mRGCs co-expressing Brn-3b and melanopsin in CFP-D2 mice revealed a significant loss of co-expression with age (Figure 7). However, there was still labeling of mRGCs with no Brn-3b staining (Figure 7B). In comparison, 8-month-old versus 14-month-old CFP-D2 mice expressed significantly decreased Brn-3b in mRGCs, however, this difference was not seen when comparing the other age groups $(P<0.01, n=4)$ (Figure 7C).

\subsection{Abnormal behavioral patterns in CFP-D2 mice}

Locomotor activity was assessed using OFT on both CFP-D2 and C57BL/6 mice of different age groups. We analyzed three major parameters in the OFT, including the total distance traveled, the number of entries into the center,
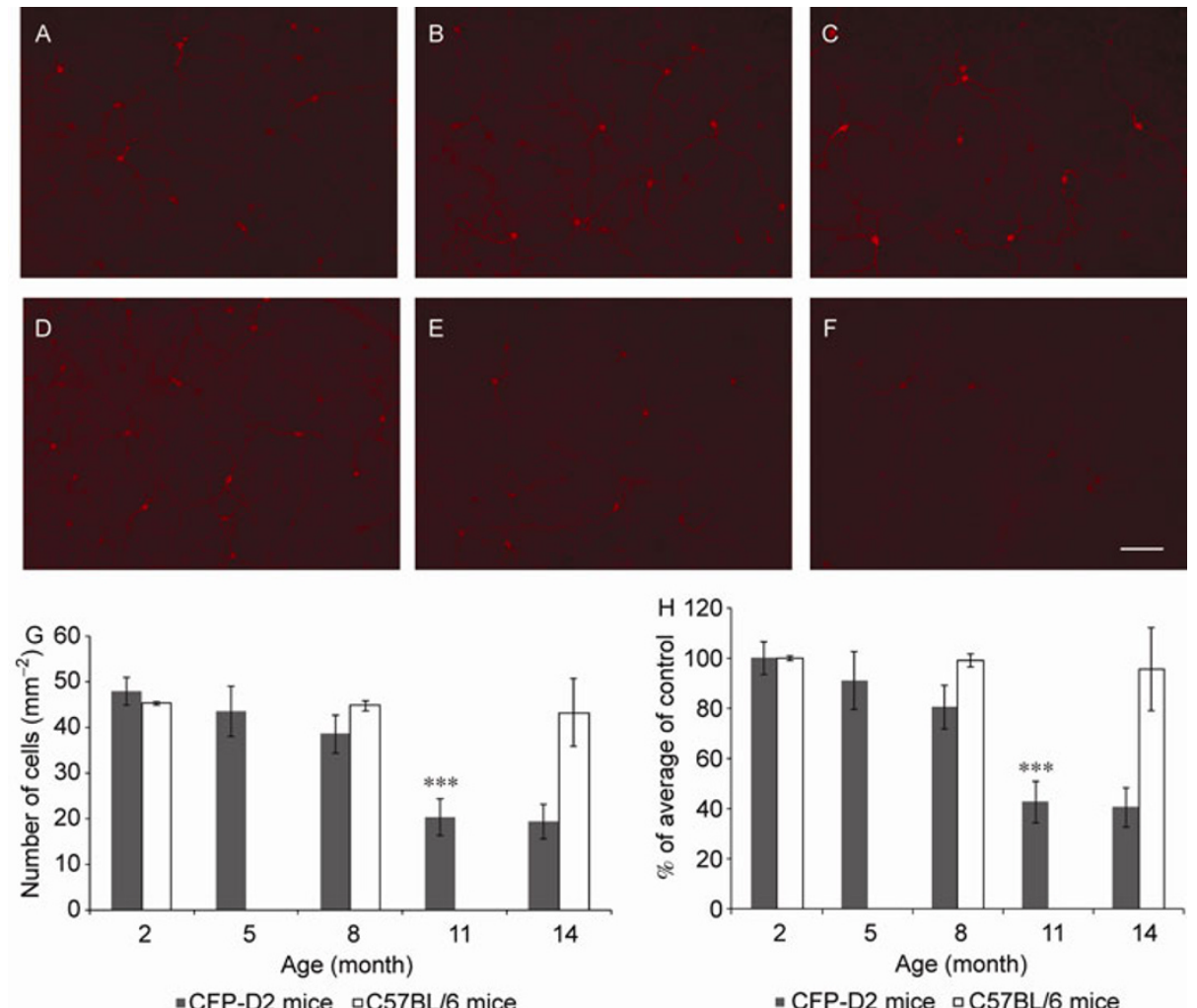

Figure 5 Density distribution patterns of mRGCs in retinas of CFP-D2 and control mice. A-C, Images captured from C57BL/6 mice of different age groups (2, 8 and 14 months old). D-F, Images captured from CFP-D2 mice of different age groups (2, 8, and 14 months old). The mRGCs were visualized by immunohistochemical staining with melanopsin antibody. G, mRGC densities in CFP-D2 and control retinas in different age groups (2 vs. 5 months old, 5 vs. 8 months old, 8 vs. 11 months old, and 11 vs. 14 months old). H, Density of mRGCs (mean \pm SD) in GCLs of both C57BL/6 and CFP-D2 mice ( $n=5$ retinas/age group). Scale bar, $100 \mu \mathrm{m}$. ***, $P<0.001$. Conventions are as shown in Figure 3. 

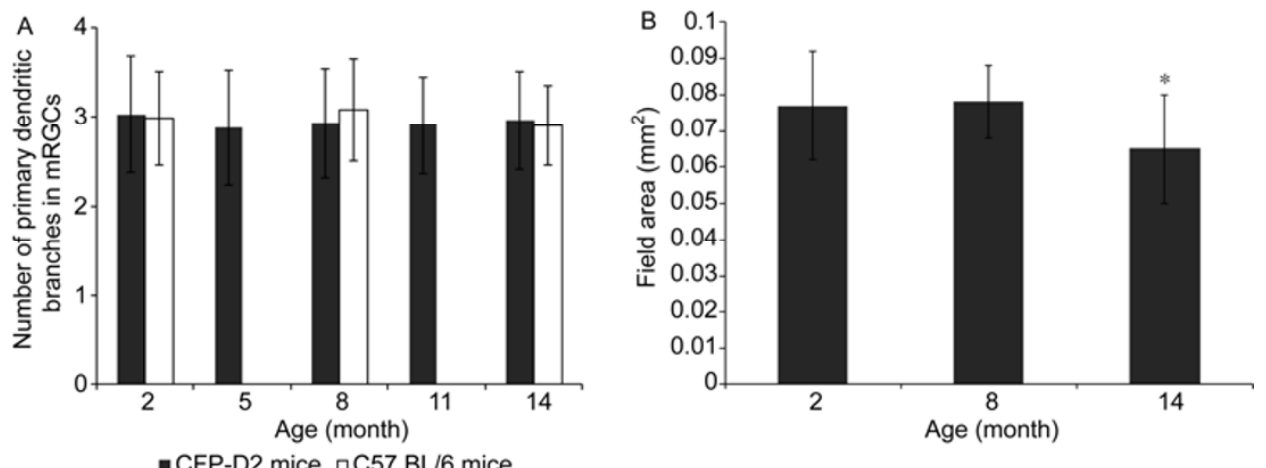

- CFP-D2 mice aC57 BL/6 mice

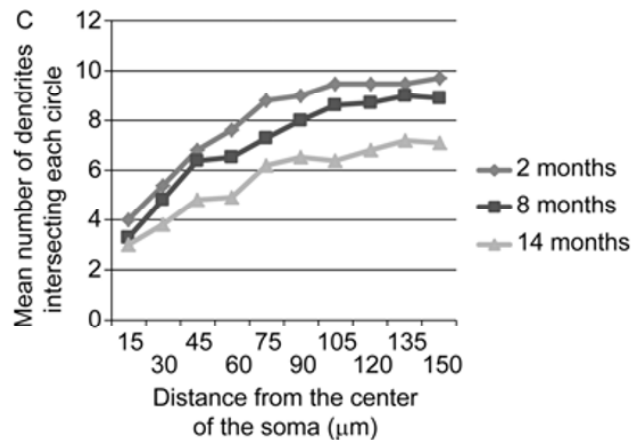

Figure 6 Histograms comparing morphological properties of mRGCs as a function of age. The comparisons of mRGCs were made between C57BL/6 and CFP-D2 mice in four age-matched groups ( 2 vs. 5 months old, 5 vs. 8 months old, 8 vs. 11 months old, and 11 vs. 14 months old, $n=10$ cells/age group). A, Histogram comparing number of primary dendritic branch points as a function of age in C57BL/6 mice (mean \pm SD). B, Histogram comparing dendritic field area as a function of age in CFP-D2 mice. C, Comparisons of the number of dendritic branches intersecting each circle among three age groups (blue, 2 months old; red, 8 months old; green, 14 months old). The abscissa designates the distance from the center of the soma ( $\mu$ m). Vertical coordinates show the average number of dendrites intersecting each circle. *, $P<0.05$.
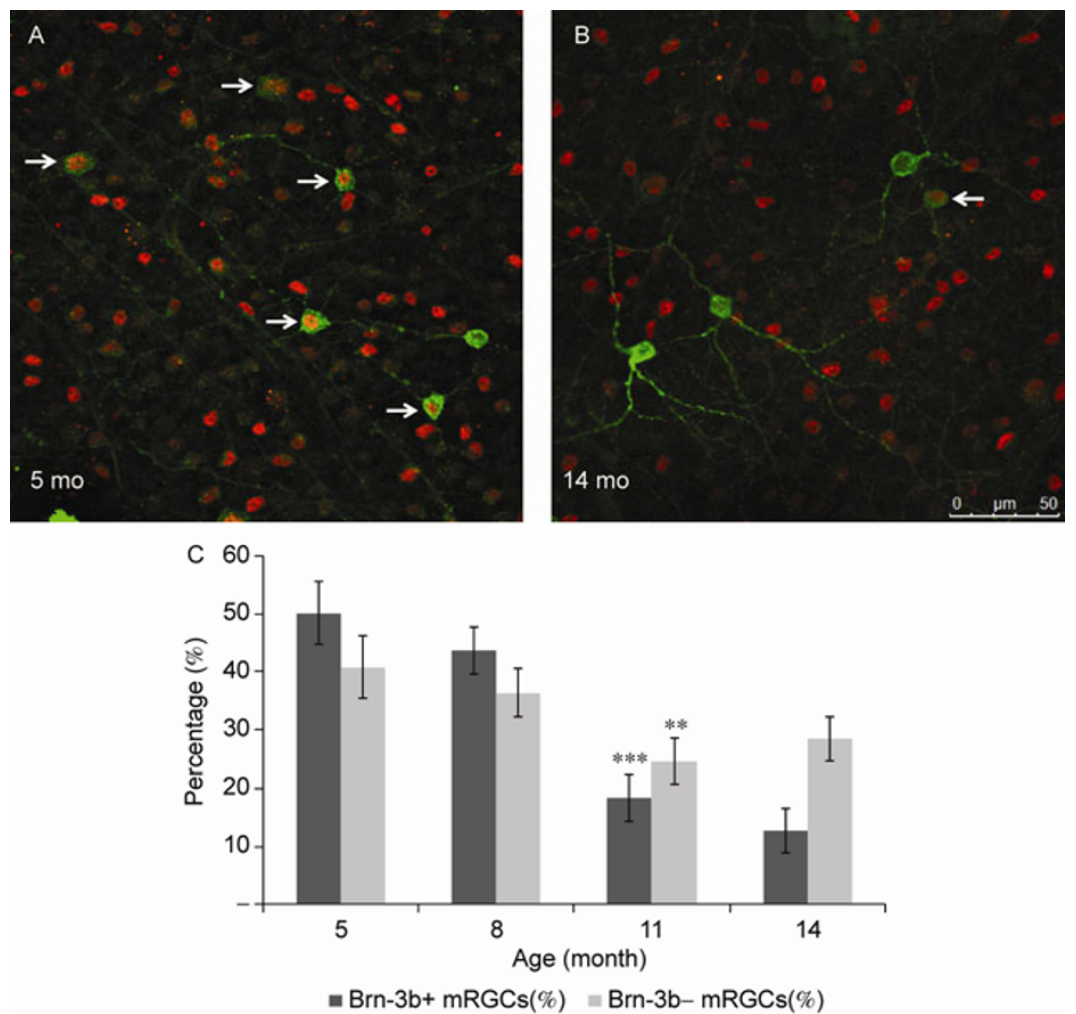

Figure 7 Reduction of Brn-3b-expressing mRGCs in CFP-D2 mice. Arrows in (A) and (B) show the doubly labeled RGCs in 5- and 14-month-old CFP-D2 mice, respectively (melanopsin in green and Brn-3b in red). The histogram (C) shows results of quantitative comparisons. Quantification analysis of mRGCs co-expressing Brn-3b and melanopsin in CFP-D2 mice shows a significant loss of co-expression with age. $* *, P<0.01 ; * * *, P<0.001$. 
and time spent in the center versus total time in the box. CFP-D2 and C57BL/6 mice demonstrated distinctive locomotor activity patterns (Figures 8 and 9). CFP-D2 mice were highly active and had a sporadic running pattern (Figure $8 \mathrm{~B}$ ), while the C57BL/6 mice traveled less and maintained regular running tracks along the sides of the box (Figure 8A). Aging C57BL/6 mice showed a progressive reduction in total distance traveled, entries into the center, and time spent in the center (Figure 9) $(P<0.05, n=5)$. However, CFP-D2 mice maintained highly active locomotor activity in all tested age groups $(2,8$, and 14 months old) (Figure 9).

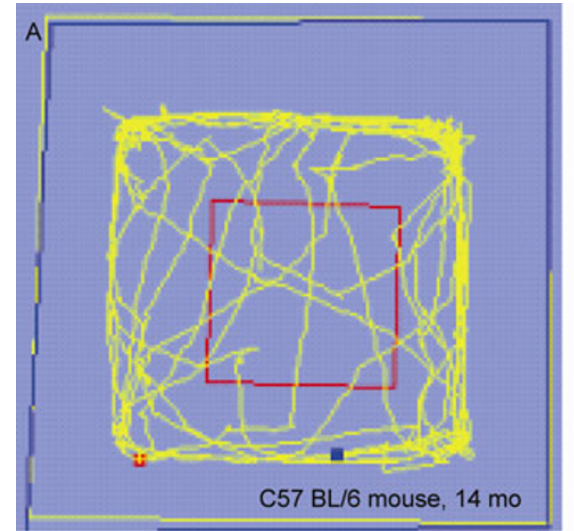

\section{Discussion}

The present study investigated the age-dependent pathologic changes of RGCs as glaucoma developed in CFP-D2 mice. The progressive elevation of IOP paralleled reduction in the average number of RGCs and mRGCs in the CFP-D2 mice. In comparison with C57BL/6 mice, CFP-D2 mice showed significantly increased locomotor activities. Thus, the present study suggests that glaucoma-induced progressive loss of RGCs, especially mRGCs degeneration, could have a substantial impact on animal behavioral response patterns.

The CFP-D2 transgenic mouse line was generated on the

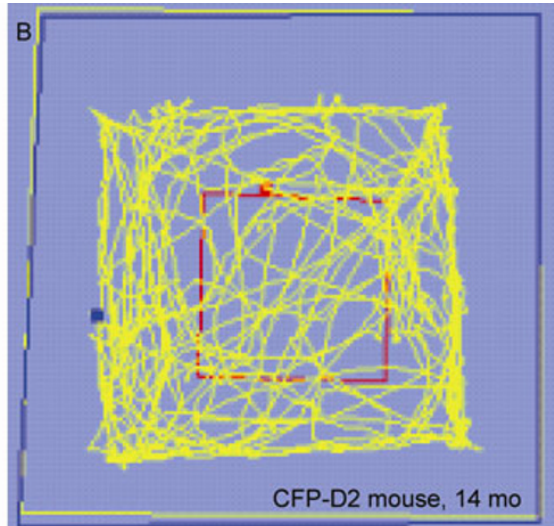

Figure 8 Locomotion tracks of C57BL/6 and CFP-D2 mice as determined by OFT. A, Tracks of locomotor activity pattern of a C57BL/6 mouse. B, Locomotor activity pattern of a CFP-D2 mouse, both at 14 months of age.
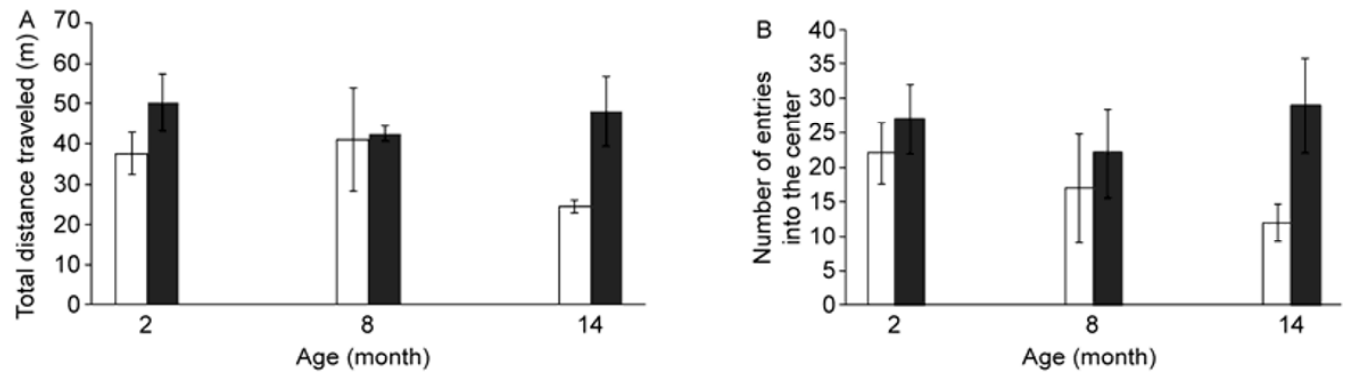

$\square$ C57BL/6 mice $\quad$ CFP-D2 mice

$\square$ C57BL/6 mice $\quad$ CFP-D2 mice

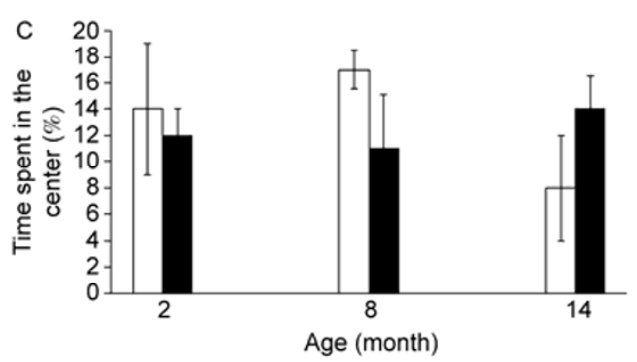

口C57BL/6 mice $\because$ CFP-D2 mice

Figure 9 Histograms comparing parameters of OFT in CFP-D2 and C57BL/6 mice as a function of age. Aging C57BL/6 mice showed a progressive reduction in total distance traveled (A), number of entries into the center (B), and time spent in the center (C). However, CFP-D2 mice maintained highly active locomotor activity in all tested age groups $(2,8$, and 14 months old $)$. 
DBA/2J background. However, unlike the conventional $\mathrm{DBA} / 2 \mathrm{~J}$ line, the RGCs in CFP-D2 retinas can be directly visualized by the expression of CFP under the Thy-1 promoter. Although CFP is not exclusively expressed in RGCs and is expressed in some cholinergic and displaced amacrine cells, misexpression is limited and minimal [35]. Using the CFP-D2 line, we investigated the general morphology of CFP-expressing RGCs at different ages. The average number of CFP-expressing RGCs decreased with age and elevated IOP. Similarly, the average number of mRGCs declined with age in CFP-D2 mice. These findings suggest that CFP can be an effective RGC marker, as well as a marker for progressive degeneration of RGCs in CFP-D2 mice.

Clinical studies have associated a high prevalence of depression with the development of glaucoma [4-14]. Similarly, progressive degeneration of mRGCs has become the most striking clinical indicator of the progression of glaucoma [36-38]. Morphological evidence shows that M1 projects to SCN and M2-5 innervate other non-visual nuclei $[16,39,40]$. The M1 cells are involved in regulation of circadian entrainment while M2-5 cells are responsible for other non-visual functions including pupillary light reflex $[16,17]$. Indeed, two early clinical studies showed that glaucoma patients suffered from afferent pupillary defects during the early stages of the disease [41], and underwent sleep disorders during later stages of the disease [6]. This implies that the non-image-forming visual pathway is disturbed or impaired in these glaucoma patients. In addition, there is substantial evidence that mRGCs play an important role in the non-image-forming visual function including behavioral mood regulation [15-18]. In agreement with these observations, the present study suggests that preferential loss of M2-5 cells could affect the animal's behavioral response patterns. We confirmed that $\mathrm{mRGCs}$ were severely affected by chronic IOP elevation in CFP-D2 mice (Figures 2 and 5-7). However, other studies have demonstrated that mRGCs were not sensitive to acute IOP increase [42]. Jakobs et al. [43] reported that 2-month-old DBA/2J mice showed proportional loss of mRGCs that was not significantly different from that of conventional RGCs. To date, however, few studies have investigated the link of RGC dysfunction, specifically the loss of mRGCs, to glaucoma induced behavioral response pattern changes or depressive behaviors. Therefore, our study provides novel and informative details regarding progressive and developmental associations between mRGC morphology, glaucoma, and behavioral activity pattern changes. Several lines of evidence demonstrate that loss of both cRGCs and mRGCs result in depression-like behavior, such as increased anxiety levels [44-47]. However, the specific neuromechanisms underlying conventional RGCs and mRGCs influence on behavioral moods remain to be investigated.

The OFT is a well-established behavioral assessment for locomotor activity and level of anxiety in rodents [48]. It has also been used in studies evaluating the effects of antidepressant treatment in anxious animals [49]. In this study, we compared locomotor activity of C57BL/6 and CFP-D2 mice at three ages (2, 8, and 14 months old). C57BL/6 mice showed reduction of locomotor activity with age, while CFP-D2 mice exhibited no statistically significant differences in total distance traveled, number of entries into the center, and time spent in the center/total time with age ( $2 \mathrm{vs}$. 8 months old or 8 vs. 14 months old). It is plausible that highly active locomotor activities are not the result of loss of conventional RGCs or mRGCs, but are caused by other factors such as genetic mutations in this species [26,27, $50,51]$. The DBA/2J mouse is a popular model for anxietyand locomotion-related behaviors [52,53], but this animal exhibits normal circadian rhythm [54]. In agreement with this finding, our results show that Brn-3b-negative mRGCs, which we suspect are M1-type mRGCs, had less injury than Brn-3b-positive mRGCs (M2-5) (Figure 7). These results suggest that abnormal behavioral response patterns could be due to injury of Brn-3b-positive mRGCs or are a compensation effect of the surviving Brn-3b-negative mRGCs. Indeed, depressive response patterns of this glaucoma mouse model can be reversed by antidepressant treatment [55]. Nevertheless, this study does not offer direct evidence to link the loss of mRGCs with the hyperactive behavior pattern observed in this glaucoma animal model. To address this question, one may consider selective elimination of mRGCs in the D2 line.

In conclusion, CFP-D2 mice have an age dependent increase in IOP and parallel morphological deterioration of mRGCs, in particular Brn-3b-positive mRGCs. Concurrently, CFP-D2 mice have hyperactive locomotor activity and sporadic locomotor patterns. Together, the present findings suggest that glaucoma induced changes in locomotor activity and behavioral patterns can be associated with dysfunctional mRGCs, most likely the Brn-3b-positive mRGCs, as observed in the CFP-D2 mouse line.

This work was supported by the National Basic Research Program of China (2009CB320900 to Pu MingLiang, 2011CB510206 to Pu MingLiang and Gao Jie), National Natural Science Foundation of China (30831160516 to Pu MingLiang), NIH EY04067 (N.C. Brecha), and VA Merit Review (N.C. Brecha). N.C. Brecha is a VA Career Research Scientist. H. Vuong was supported by a summer fellowship from the PKU-UCLA Joint Research Institute.

1 Quigley H A. Number of people with glaucoma worldwide. Br J Ophthalmol, 1996, 80: 389-393

2 Quigley H A, Broman A T. The number of people with glaucoma worldwide in 2010 and 2020. Br J Ophthalmol, 2006, 90: 262-267

3 Wong A A, Brown R E. A neurobehavioral analysis of the prevention of visual impairment in the DBA/2J mouse model of glaucoma. Invest Ophthalmol Vis Sci, 2012, 53: 5956-5966

4 Jayawant S S, Bhosle M J, Anderson R T, et al. Depressive symptomatology, medication persistence, and associated healthcare costs in older adults with glaucoma. J Glaucoma, 2007, 16: 513-520

5 Pappa C, Hyphantis T, Pappa S, et al. Psychiatric manifestations and 
personality traits associated with compliance with glaucoma treatment. J Psychosom Res, 2006, 61: 609-617

6 Onen S H, Mouriaux F, Berramdane L, et al. High prevalence of sleep-disordered breathing in patients with primary open-angle glaucoma. Acta Ophthalmol Scan, 2000, 78: 638-641

7 Kohn A N, Moss A P, Podos S M. Relative afferent pupillary defects in glaucoma without characteristic field loss. Arch Ophthalmol, 1979, 97: 294-296

8 Warthen D M, Wiltgen B J, Provencio I. Light enhances learned fear. Proc Natl Acad Sci USA, 2011, 108: 13788-13793

9 Roecklein K A, Wong P M, Miller M A, et al. Melanopsin, photosensitive ganglion cells, and seasonal affective disorder. Neurosci Biobehav Rev, 2013, 37: 229-239

10 Cumurcu T, Cumurcu B E, Celikel F C, et al. Depression and anxiety in patients with pseudoexfoliative glaucoma. Gen Hosp Psychiatry, 2006, 28: 509-515

11 Jampel H D, Frick K D, Janz N K, et al. Depression and mood indicators in newly diagnosed glaucoma patients. Am J Ophthalmol, 2007, 144: 238-244

12 Yochim B P, Mueller A E, Kane K D, et al. Prevalence of cognitive impairment, depression, and anxiety symptoms among older adults with glaucoma. J Glaucoma, 2012, 21: 250-254

13 Wang S Y, Singh K, Lin S C. Prevalence and predictors of depression among participants with glaucoma in a nationally representative population sample. Am J Ophthalmol, 2012, 154: 436-444

14 Popescu M L, Boisjoly H, Schmaltz H, et al. Explaining the relationship between three eye diseases and depressive symptoms in older adults. Invest Ophthalmol Vis Sci, 2012, 53: 2308-2313

15 Do M T, Yau K W. Intrinsically photosensitive retinal ganglion cells. Physio Rev, 2010, 90: 1547-1581

16 Chen S K, Badea T C, Hattar S. Photoentrainment and pupillary light reflex are mediated by distinct populations of ipRGCs. Nature, 2011, 476: 92-95

17 Lucas R J, Hattar S, Takao M, et al. Diminished pupillary light reflex at high irradiances in melanopsin-knockout mice. Science, 2003, 299: 245-247

18 LeGates T A, Altimus C M, Wang H, et al. Aberrant light directly impairs mood and learning through melanopsin-expressing neurons. Nature, 2012, 491: 594-598

19 Ren C, Luan L, Lau B W, et al. Direct retino-raphe projection alters serotonergic tone and affective behavior. Neuropsychopharmacology, 2013, doi: 10.1038/npp.2013.35

20 Allcutt D, Berry M, Sievers J. A qualitative comparison of the reactions of retinal ganglion cell axons to optic nerve crush in neonatal and adult mice. Brain Res, 1984, 318: 231-240

21 Kielczewski J L, Pease M E, Quigley H A. The effect of experimental glaucoma and optic nerve transection on amacrine cells in the rat retina. Invest Ophthalmol Vis Sci, 2005, 46: 3188-3196

22 Villegas-perez M P, Salvador-silva M, Ruiz-gomez J M, et al. Retinal ganglion cell death after different transient periods of pressureinduced ischemia and survival intervals. A quantitative in vivo study. Invest Ophthalmol Vis Sci, 2002, 37: 2002-2014

23 Yu S, Tanabe T, Yoshimura N. A rat model of glaucoma induced by episcleral vein ligation. Exp Eye Res, 2006, 83: 758-770

24 Nair K S, Hmani-Aifa M, Ali Z, et al. Alteration of the serine protease PRSS56 causes angle-closure glaucoma in mice and posterior microphthalmia in humans and mice. Nat Genet, 2011, 43: 579584

25 John S W, Smith R S, Savinova O V, et al. Essential iris atrophy, pigment dispersion, and glaucoma in DBA/2J mice. Invest Ophthalmol Vis Sci, 1998, 39: 951-962

26 Chang B, Smith R S, Hawes N L, et al. Interacting loci cause severe iris atrophy and glaucoma in DBA/2J mice. Nature Genet, 1999, 21: 405-409

27 Anderson M G, Smith R S, Hawes N L, et al. Mutations in genes encoding melanosomal proteins cause pigmentary glaucoma in DBA/2J mice. Nature Genet, 2002, 30: 81-85

28 Feng G, Mellor R H, Bernstein M, et al. Imaging neuronal subsets in transgenic mice expressing multiple spectral variants of GFP. Neuron,
2000, 28: 41-51

29 Raymond I D, Pool A L, Vila A, et al. A Thy1-CFP DBA/2J mouse line with cyan fluorescent protein expression in retinal ganglion cells. Vis Neurosci, 2009, 26: 453-465

30 Luan L, Ren C, Lau B W, et al. Y-like retinal ganglion cells innervate the dorsal raphe nucleus in the Mongolian gerbil (Meriones unguiculatus). PLoS ONE, 2011, 6: e18938

31 Bouwknecht J A, Paylor R. Behavioral and physiological mouse assays for anxiety: a survey in nine mouse strains. Behav Brain Res, 2002, 136: 489-501

32 Leahy K M, Ornberg R L, Wang Y, et al. Quantitative ex vivo detection of rodent retinal ganglion cells by immunolabeling Brn-3b. Exp Eye Res, 2004, 79: 131-140

33 Goz D, Studholme K, Lappi D A, et al. Targeted destruction of photosensitive retinal ganglion cells with a saporin conjugate alters the effects of light on mouse circadian rhythms. PLoS ONE, 2008, 3: e3153

34 Sholl D A. Dendritic organization in the neurons of the visual and motor cortices of the cat. J Anat, 1953, 87: 387-406

35 Raymond I D, Vila A, Huynh U C, et al. CFP expression in ganglion and amacrine cells in a thy1-CFP transgenic mouse retina. Mol Vis, 2008, 14: 1559-1574

36 Feigl B, Mattes D, Thomas R, et al. Intrinsically photosensitive (melanopsin) retinal ganglion cell function in glaucoma. Invest Ophthalmol Vis Sci, 2011, 52: 4362-4367

37 Kankipati L, Girkin C A, Gamlin P D. The post-illumination pupil response is reduced in glaucoma patients. Invest Ophthalmol Vis Sci, 2011, 52: 2287-2292

38 La Morgia C, Ross-Cisneros F N, Hannibal J, et al. Melanopsinexpressing retinal ganglion cells: implications for human diseases. Vision Res, 2011, 51: 296-302

39 Hattar S, Kumar M, Park A, et al. Central projections of melanopsin-expressing retinal ganglion cells in the mouse. J Comp Neurol, 2006, 497: 326-349

40 Xiang M, Zhou L, Macke J P, et al. The Brn-3 family of POU-domain factors: primary structure, binding specificity, and expression in subsets of retinal ganglion cells and somatosensory neurons. J Neurosci, 1995, 15: 4762-4785

41 Kaback M B, Burde R M, Becker B. Relative afferent pupillary defect in glaucoma. Am J Ophthalmol, 1976, 81: 462-468

42 Li R S, Chen B Y, Tay D K, et al. Melanopsin-expressing retinal ganglion cells are more injury-resistant in a chronic ocular hypertension model. Invest Ophthalmol Vis Sci, 2006, 47: 2951-2958

43 Jakobs T C, Libby R T, Ben Y, et al. Retinal ganglion cell degeneration is topological but not cell type specific in DBA/2J mice. J Cell Biol, 2005, 171: 313-325

44 Dulawa S C, Holick K A, Gundersen B, et al. Effects of chronic fluoxetine in animal models of anxiety and depression. Neuropsychopharmacology, 2004, 29: 1321-1330

45 Weiss G A, Goldich Y, Bartov E, et al. Compliance with eye care in glaucoma patients with comorbid depression. IMAJ, 2011, 13: 730734

46 Hollo G, Kothy P, Anna G, et al. Personality traits, depression, and objectively measured adherence to once-daily prostaglandin analog medication in glaucoma. J Glaucoma, 2009, 18: 288-292

47 Wilson M R, Coleman A L, Yu F, et al. Depression in patients with glaucoma as measured by self-report surveys. Ophthalmology, 2002, 109: 1018-1022

48 Lipkind D, Sakov A, Kafkafi N, et al. New replicable anxiety-related measures of wall vs center behavior of mice in the open field. J Appl Physiol, 2004, 97: 347-359

49 Can A, Blackwell R A, Piantadosi S C, et al. Antidepressant-like responses to lithium in genetically diverse mouse strains. Genes Brain Behav, 2011, 10: 434-443

50 Roecklein K A, Rohan K J, Duncan W C, et al. A missense variant (P10L) of the melanopsin (OPN4) gene in seasonal affective disorder. J Affect Disord, 2009, 114: 279-285

51 Jawahar M C, Brodnicki T C, Quirk F, et al. Behavioral analysis of congenic mouse strains confirms stress-responsive Loci on chromo- 
somes 1 and 12. Behav Genet, 2008, 38: 407-416

52 Sokoloff G, Parker C C, Lim J E, et al. Anxiety and fear in a cross of C57BL/6J and DBA/2J mice: mapping overlapping and independent QTL for related traits. Genes Brain Behav, 2011, 10: 604-614

53 Fraser L M, Brown R E, Hussin A, et al. Measuring anxiety- and locomotion-related behaviors in mice: a new way of using old tests.
Psychopharmacology (Berl), 2010, 211: 99-112

54 Hofstetter J R, Hofstetter A R, Hughes A M, et al. Intermittent long-wavelength red light increases the period of daily locomotor activity in mice. J Circadian Rhythms, 2005, 3: 8-15

55 Hovatta I, Tennant R S, Helton R, et al. Glyoxalase 1 and glutathione reductase 1 regulate anxiety in mice. Nature, 2005, 438: 662-666

Open Access This article is distributed under the terms of the Creative Commons Attribution License which permits any use, distribution, and reproduction in any medium, provided the original author(s) and source are credited. 